\title{
Fractional Delivery: Toward a Painless, Safe and Effective Cutaneous Vaccination
}

\author{
Ji Wang* \\ Department of Dermatology, Harvard Medical School, 50 Blossom Street, Edwards 211, Boston, USA.
}

*Corresponding author: Wang Ji, Department of Dermatology, Harvard Medical School, 50 Blossom Street, Edwards 211, Boston, MA 02114, USA, Tel: 617-724-2653; E-mail: Jwang10@mgh.harvard.edu

Received date: January 18, 2017; Accepted date: January 19, 2017; Published date: January 24, 2017

Copyright: (c) 2017 Wang J. This is an open-access article distributed under the terms of the Creative Commons Attribution License, which permits unrestricted use, distribution, and reproduction in any medium, provided the original author and source are credited

\section{Editorial}

Delivering vaccines into skin (Cutaneous Vaccination) is one of most promising strategies for the next generation vaccination. Cutaneous vaccination enables high vaccination efficacy harnessing the unique immunological nature of skin that numerous antigen presenting cells (APCs) reside in epidermis and dermis concomitant with an abundant network of lymphatic vessels. In sharp contrast, as a conventional vaccination site for most vaccines, muscle has few APCs. Therefore, cutaneous vaccination always showed a superior efficacy to intramuscular injection for a number of vaccines, including Influenza, Hepatitis B, Rabies, Polio etc. This advantage enables us to address the problem of age-related low vaccination efficacy in very young $(<2$ years) and elder ( $>65$ years) populations, to overcome vaccine shortage in developing countries (e.g. inactivated poliovirus vaccine) or during a pandemic (e.g. pandemic influenza vaccine) by sparing antigen doses.

However, the clinic application of cutaneous vaccination is hindered by limitations of current intradermal injection technology. First, intradermal injection via hypodermic needles, also known as the Mantoux injection method, can only be properly handled by experienced professionals. Second, injection of a large volume $(>100$ $\mu \mathrm{l})$ into skin induces more pain than intramuscular injection, resulting in poor patient compliance. Third and most importantly, skin is prone to inducing inflammations. As a result, conventional intradermal injection via hypodermic needles always induced a higher level of local reactions demonstrated by a number of clinic studies. For example, intradermal injection of influenza vaccines always induced erythema and swelling, persistent for days. Therefore, new technologies and strategies are needed to fully realize the potential of cutaneous vaccination.

Fractional delivery is a promising strategy for cutaneous vaccination. It could be accomplished by microneedle arrays, fractional laser-mediated delivery methods or hollow microneedle arrays. Vaccines and adjuvants are delivered into hundreds of tiny spots, usually smaller than $300 \mu \mathrm{m}$ in diameter, instead of injecting all antigens into one big spot. These tiny spots are well separated and surrounded by healthy tissues. The tiny injection site with fractional antigen dose reduces the local irritations induced by vaccines or adjuvants and ensures a quick recovery of injured tissues without compromising vaccination efficacies. Moreover, the distance between each spot also matters. A longer distance could further constrain local reactions [1]. However, the optimal distance should be determined individually for each vaccine and delivery system.

Factional delivery of Bacillus Calmette-Guérin (BCG) vaccine via a biodegradable microneedle array resulted in a significantly improved skin condition compared to the conventional intradermal injection which always induces severe skin lesions and permanent scars in human skin [1]. A similar result has been observed from the influenza vaccine study. Cutaneous vaccination of inactivated influenza virus vaccine by microneedles showed mild to no local reactions in swine, a clinic relevant model for human skin [1]. Besides live bacteria vaccines (BCG) and inactivated virus vaccines (Influenza), vaccination of liveattenuated viral vectors could also benefit from fractional delivery. For example, delivery vaccinia virus, the vaccine for smallpox, by lasermediated micro-fractional powder delivery induced fewer skin lesions with greatly accelerated skin recovery [2].

Fractional delivery is not only beneficial for delivering vaccines into skin, but also improves the safety of intradermal delivery of adjuvants. Adjuvant is a very important component of modern vaccines. They are able to augment the efficacy of vaccines, accelerate the induction of immune responses, prolong the duration of immunity and switch the type of immune responses. However, most adjuvants that have been approved or are under development induce severe skin reactions [3]. Fractional delivery may address this issue. A combination of MPL and $\mathrm{CpG}$ adjuvants induces severe skin irritations. However, if MPL/CpG is fractionally delivered, the skin irritations could be significantly impaired [3]. Microneedles-mediated fractionally delivery has been used to deliver QS-21 and Alum adjuvants into skin [4,5]. Previously, alum adjuvant could only be used for intramuscular injections because of its potential to induce skin reactions. Moreover, a laser based adjuvant that fractionally delivers micro-sterile inflammation into skin also showed good safety profiles [6].

Taken together, fractional delivery of vaccines or adjuvants retains the efficacy of cutaneous vaccination but significantly improves the safety. In additional, the fractional delivery technologies such as microneedles induce less pain than conventional intradermal injection, because of their small needle size. Therefore, fractional delivery of vaccines into skin is a promising strategy for a painless, safe and effective vaccination. Expanding this strategy to a broader spectrum of vaccines/adjuvants and developing new technologies are expected. Further clinical trials are merited.

\section{References}

1. Wang J, Li B, Wu MX (2015) Effective and lesion-free cutaneous influenza vaccination. Proc Natl Acad Sci USA 112: 5005-5010.

2. Chen X, Kositratna G, Zhou C, Manstein D, Wu MX (2014) Microfractional epidermal powder delivery for improved skin vaccination. J Control Release 192: 310-316.

3. Chen X, Wang J, Shah D, Wu MX (2013) An update on the use of laser technology in skin vaccination. Expert Rev Vaccines 12: 1313-1323. 
Citation: Wang J (2017) Fractional Delivery: Toward a Painless, Safe and Effective Cutaneous Vaccination. J Vaccines Vaccin 8: e130. doi: $10.4172 / 2157-7560.1000 \mathrm{e} 130$

Page 2 of 2

4. Ng HI, Fernando GJ, Depelsenaire AC, Kendall MA (2016) Potent response of QS-21 as a vaccine adjuvant in the skin when delivered with the Nanopatch, resulted in adjuvant dose sparing. Sci Rep 6: 29368.

5. Corbett HJ, Fernando GJ, Chen X, Frazer IH, Kendall MA (2010) Skin vaccination against cervical cancer associated human papillomavirus with a novel micro-projection array in a mouse model. PLoS One 5: e13460.
6. Wang J, Shah D, Chen X, Anderson RR, Wu MX (2014) A micro-sterile inflammation array as an adjuvant for influenza vaccines. Nat Commun 5: 4447. 